\title{
Strong Law of Large Numbers for Countable Markov Chains Indexed by an Infinite Tree with Uniformly Bounded Degree
}

\author{
Bao Wang, ${ }^{1,2}$ Weiguo Yang, ${ }^{1}$ Zhiyan Shi, ${ }^{1}$ and Qingpei Zang ${ }^{3}$ \\ ${ }^{1}$ Faculty of Science, Jiangsu University, Zhenjiang 212013, China \\ ${ }^{2}$ College of Mathematics and Physics, Xuzhou Institute of Technology, Xuzhou 221000, China \\ ${ }^{3}$ School of Mathematical Science, Huaiyin Normal University, Huaian 223300, China \\ Correspondence should be addressed to Bao Wang; wangbao@xzit.edu.cn
}

Received 2 October 2013; Revised 15 January 2014; Accepted 3 February 2014; Published 13 March 2014

Academic Editor: Martin Ostoja-Starzewski

Copyright ( 2014 Bao Wang et al. This is an open access article distributed under the Creative Commons Attribution License, which permits unrestricted use, distribution, and reproduction in any medium, provided the original work is properly cited.

We study the strong law of large numbers for the frequencies of occurrence of states and ordered couples of states for countable Markov chains indexed by an infinite tree with uniformly bounded degree, which extends the corresponding results of countable Markov chains indexed by a Cayley tree and generalizes the relative results of finite Markov chains indexed by a uniformly bounded tree.

\section{Introduction}

A tree $T$ is a graph which is connected and contains no circuits. Given any two vertices $\alpha \neq \beta \in T$. Let $\overline{\alpha \beta}$ be the unique path connecting $\alpha$ and $\beta$. Define the distance $d(\alpha, \beta)$ to be the number of edges contained in the path $\overline{\alpha \beta}$

Select a vertex as the root (denoted by $o$ ). For any two vertices $\sigma$ and $t$ of tree $T$, we write $\sigma \leq t$ if $\sigma$ is on the unique path from the root $o$ to $t$. We denote by $\sigma \wedge t$ the vertex farthest from $o$ satisfying $\sigma \wedge t \leq t$ and $\sigma \wedge t \leq \sigma$. For any vertex $t$ of tree $T$, we denote by $|t|$ the distance between $o$ and $t$. The set of all vertices with distance $n$ from the root $o$ is called the $n$th level of $T$. For any vertex $t$ of tree $T$, we denote the predecessor of $t$ by $1_{t}$, the predecessor of $1_{t}$ by $2_{t}$, and the predecessor of $(n-1)_{t}$ by $n_{t}$. We also call $n_{t}$ the $n$th predecessor of $t$. Similarly, we denote the one of the successor of $t$ by $1^{t}$, the one of the successors of $1^{t}$ by $2^{t}$, and one of the successors of $(n-1)^{t}$ by $n^{t}$. We denote by $T^{(n)}$ the subtree comprised of level 0 (the root $o$ ) through level $n$, and by $L_{n}$ the set of all vertices on level $n$. In this paper, we mainly consider an infinite tree which has uniformly bounded degrees. That is, the numbers of neighbors of any vertices in this tree are uniformly bounded; we call it the uniformly bounded tree. If the root of a tree has $M$ neighboring vertices and other vertices have $M+1$ neighboring vertices, we call this type of tree a Cayley tree and denote it by $T_{C, M}$. It is easy to see that this type of tree is the special case of uniformly bounded tree. Let $S$ be the subgraph of $T, X^{S}=\left\{X_{t}, t \in S\right\}$, and $x^{S}$ the realization of $X^{S}$. We denote by $|S|$ the number of vertices of $S$.

Definition 1 (see [1]). Let $T$ be a local finite and infinite tree; that is, the tree has infinite vertices and the degrees of any vertices in this tree are finite. Let $G=\{0,1,2, \ldots\}$ be a countable state space and $\left\{X_{t}, t \in T\right\}$ a collection of $G$-valued random variables defined on the probability space $(\Omega, \mathscr{F}, \mathbf{P})$. Let

$$
p=(p(x), x \in G)
$$

be a distribution on $G$, and let

$$
P=(P(y \mid x)), \quad x, y \in G
$$


be a stochastic matrix on $G^{2}$. Let $n$ be any positive integer. If for any vertices $t, t_{1}, t_{2}, \ldots t_{n} \in T, t_{i} \wedge t \leq 1_{t}, 1 \leq i \leq n$,

$$
\begin{gathered}
\mathbf{P}\left(X_{t}=y \mid X_{1_{t}}=x \text { and } X_{t_{1}}=x_{t_{1}}, X_{t_{2}}=x_{t_{2}}, \ldots, X_{t_{n}}=x_{t_{n}}\right) \\
=\mathbf{P}\left(X_{t}=y \mid X_{1_{t}}=x\right)=P(y \mid x), \quad \forall x, y \in G \\
\mathbf{P}\left(X_{o}=x\right)=p(x), \quad \forall x \in G
\end{gathered}
$$

$\left\{X_{t}, t \in T\right\}$ will be called $G$-valued Markov chains indexed by this tree $T$ with the initial distribution (1) and the transition matrix (2) or called $T$-indexed Markov chains with state space G.

Definition 2 (see [2, page 157]). Let $P$ be a stochastic matrix defined on the countable state space $G$. If there exists a distribution $\pi=(\pi(0), \pi(1), \ldots)$ satisfying

$$
\sup _{i} \sum_{j}\left|P^{(n)}(j \mid i)-\pi(j)\right| \longrightarrow 0, \quad \text { as } n \longrightarrow \infty \text {, }
$$

where $P^{(n)}(j \mid i)$ is the $n$-step transition probability determined by $P$, then $P$ is said to be strongly ergodic with distribution $\pi$. Obviously, if (5) holds, then we have $\pi P=\pi$, and $\pi$ is said to be the stationary distribution determined by $P$.

Let $\left\{X_{n}, n \geq 0\right\}$ be a sequence of random variables taking values in state space $S=\{1,2, \ldots, N\}$ with the joint distribution

$$
\begin{array}{r}
p\left(x_{0}, \ldots, x_{n}\right)=\mathbf{P}\left(X_{0}=x_{0}, \ldots, X_{n}=x_{n}\right), \\
x_{i} \in S, 0 \leq i \leq n, n \geq 0 .
\end{array}
$$

Let

$$
f_{n}(\omega)=-\frac{1}{n} \ln p\left(X_{0}, \ldots, X_{n}\right)
$$

$f_{n}(\omega)$ is called the entropy density of $\left\{X_{i}, 0 \leq i \leq n\right\}$.

The convergence of $f_{n}(\omega)$ to a constant in a sense $\left(L_{1}\right.$ convergence, convergence in probability, and a.e. convergence) is called Shannon-McMillan-Breiman theorem or asymptotic equipartition property (AEP) in information theory. Shannon [3] first proved AEP in convergence in probability for finite stationary ergodic sequence of random variables. McMillan [4] and Breiman [5, 6] proved AEP in $L_{1}$ and a.e. convergence, respectively, for finite stationary ergodic sequence of random variables. Chung [7] generalized Breiman's result to countable case.

The subject of tree-indexed processes is rather young. Benjamini and Peres [1] have given the notion of the treeindexed Markov chains and studied the recurrence and rayrecurrence for them. Guyon [8] has given the definition of bifurcating Markov chains indexed by binary tree and studied their limit theorems. Berger and Ye [9] have studied the existence of entropy rate for some stationary random fields on a homogeneous tree. Ye and Berger $[10,11]$ by using Pemantle's result [12] and a combinatorial approach have studied the

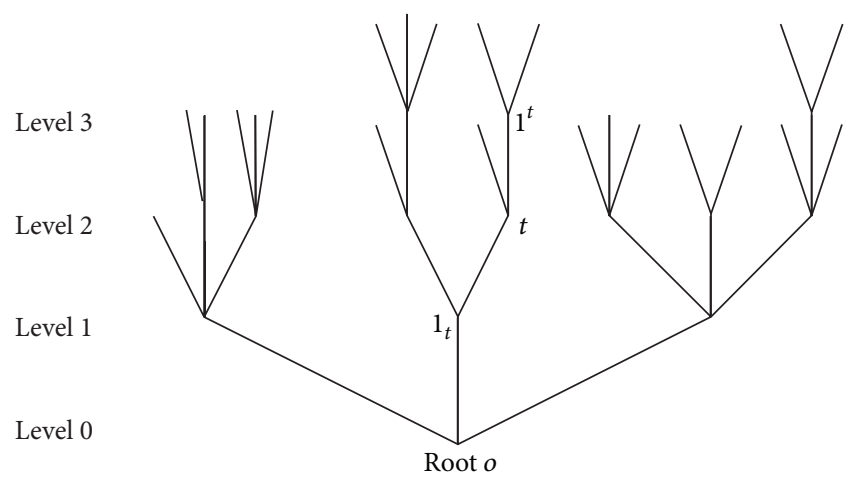

FIGURE 1: Uniformly bounded tree $T$.

asymptotic equipartition property (AEP) in the sense of convergence in probability for a PPG-invariant and ergodic random field on a homogeneous tree. Yang [13] has studied the strong law of large numbers and the asymptotic equipartition property (AEP) for finite Markov chains indexed by a homogeneous tree. Yang and Ye [14] have studied the strong law of large numbers and the asymptotic equipartition property (AEP) for finite level-nonhomogeneous Markov chains indexed by a homogeneous tree. Huang and Yang [15] have studied the strong law of large numbers and the asymptotic equipartition property (AEP) for finite Markov chains indexed by an infinite tree with uniformly bounded degree. Recently, Wang et al. [16] have studied the strong law of large numbers for countable Markov chains indexed by a Cayley tree.

In some previous articles, only the tree-indexed Markov chains with the finite state space are considered; meanwhile the countable case has very important theoretical significance, so Chung [7] generalized Breiman's result $[5,6]$ to the countable case. Wang et al. [16] have studied the strong law of large numbers countable Markov chains indexed by a Cayley tree.

The technique used to study the strong law of large numbers for countable Markov chains indexed by trees is different from that for finite case. The processing method of finite state space cannot apply to countable state space, because the sum and limit cannot be exchanged. For studying the strong law of large numbers for countable Markov chains indexed by trees, we first establish a strong limit theorem then use this strong limit theorem and smoothing property of conditional expectation repeatedly to establish our strong law of large numbers. In this paper, we use the same approach used in [16] to study the strong law of large numbers for Markov chains indexed by a uniformly bounded tree. Our results generalize the results of Huang and Yang [15] for finite Markov chains indexed by a uniformly bounded tree (Figure 1) and the results of Wang et al. [16] for countable Markov chains indexed by a Cayley tree.

\section{Some Lemmas}

Before proving the main results, we begin with some lemmas. 
Lemma 3. Let $T$ be an infinite tree with uniformly bounded degree, $\left\{X_{t}, t \in T\right\}$ a T-indexed Markov chain with countable state space $G$ defined as before, and $\left\{g_{t}(x, y), t \in T\right\}$ uniformly bounded functions defined on $G^{2}$. Let

$$
\begin{gathered}
G_{n}(\omega)=\sum_{t \in T^{(n)} \backslash\{o\}} E\left[g_{t}\left(X_{1_{t}}, X_{t}\right) \mid X_{1_{t}}\right], \\
H_{n}(\omega)=\sum_{t \in T^{(n)} \backslash\{o\}} g_{t}\left(X_{1_{t}}, X_{t}\right) .
\end{gathered}
$$

Then for all $N \geq 0$, we have

$$
\lim _{n \rightarrow \infty} \frac{H_{n}(\omega)-G_{n}(\omega)}{\left|T^{(n+N)}\right|}=0 \quad \text { a.e. }
$$

Proof. Huang and Yang (see [15, Theorem 1]) have obtained a similar result for finite Markov chains indexed by a uniformly bounded tree. By checking carefully the proof of that theorem, one can find it also holds for countable Markov chains indexed by a uniformly bounded tree, so the proof of this lemma is omitted.

Lemma 4. Let $T$ and $\left\{X_{t}, t \in T\right\}$ be defined as Lemma 3, $\mathscr{F}_{n}=$ $\sigma\left(X^{T^{(n)}}\right)$, as $t \in L_{n}, n \geq 1, \forall k \in G, \forall h \geq 1$, and we have

$$
\mathbf{P}\left(X_{h^{t}}=k \mid \mathscr{F}_{n}\right)=\mathbf{P}\left(X_{h^{t}}=k \mid X_{t}\right) .
$$

Proof. We only need to prove the situation of $h=2$. By the Markov property (3), we have

$$
\begin{aligned}
\mathbf{P} & \left(X_{2^{t}}=k \mid \mathscr{F}_{n}\right) \\
& =\sum_{m} \mathbf{P}\left(X_{2^{t}}=k, X_{1^{t}}=m \mid \mathscr{F}_{n}\right) \\
& =\sum_{m} \mathbf{P}\left(X_{2^{t}}=k \mid X_{1^{t}}=m, \mathscr{F}_{n}\right) \mathbf{P}\left(X_{1^{t}}=m \mid \mathscr{F}_{n}\right) \\
& =\sum_{m} \mathbf{P}\left(X_{2^{t}}=k \mid X_{1^{t}}=m, X_{t}\right) \mathbf{P}\left(X_{1^{t}}=m \mid X_{t}\right) \\
& =\sum_{m} \mathbf{P}\left(X_{2^{t}}=k, X_{1^{t}}=m \mid X_{t}\right)=\mathbf{P}\left(X_{2^{t}}=k \mid X_{t}\right) .
\end{aligned}
$$

By induction, (10) holds for $h \geq 2$.

\section{Strong Law of Large Numbers}

In the following, let $N \geq 0, k \in G, d^{0}(t)=1$, let $N_{\tau}$ be the $N$ th predecessor of $\tau$ defined as before, and

$$
\begin{gathered}
d^{N}(t)=\left|\tau \in T: N_{\tau}=t\right|, \quad S_{k}^{N}(A)=\sum_{t \in A} I_{k}\left(X_{t}\right) d^{N}(t) \\
S_{l, k}^{N}(A)=\sum_{t \in A} I_{l}\left(X_{1_{t}}\right) I_{k}\left(X_{t}\right) d^{N}(t)
\end{gathered}
$$

where

$$
I_{k}(i)= \begin{cases}1, & i=k, \\ 0, & i \neq k .\end{cases}
$$

Theorem 5. Let $T$ be an infinite tree with uniformly bounded degree, $P$ a strongly ergodic stochastic matrix, and $\pi$ the unique stationary distribution of $P$. Let $\left\{X_{t}, t \in T\right\}$ be a T-indexed Markov chain taking values in countable state space $G$ with the stochastic matrix $P$. Then, for any integer $N \geq 0$, we have

$$
\lim _{n \rightarrow \infty} \frac{S_{k}^{N}\left(T^{(n)}\right)}{\left|T^{(n+N)}\right|}=\pi(k) \quad \text { a.e., } k \in G \text {. }
$$

Proof. Let $g_{t}(x, y)=d^{N}(t) I_{k}(y)$ for all $t \in T$ in Lemma 3; then by (8) and (12), we have

$$
\begin{aligned}
H_{n}(\omega)= & \sum_{t \in T^{(n)} \backslash\{o\}} d^{N}(t) I_{k}\left(X_{t}\right)=S_{k}^{N}\left(T^{(n)}\right)-d^{N}(o) I_{k}\left(X_{o}\right) \\
G_{n}(\omega) & =\sum_{t \in T^{(n)} \backslash\{o\}} E\left[d^{N}(t) I_{k}\left(X_{t}\right) \mid X_{1_{t}}\right] \\
& =\sum_{t \in T^{(n)} \backslash\{o\}} d^{N}(t) \mathbf{P}\left(X_{t}=k \mid X_{1_{t}}\right) \\
& =\sum_{t \in T^{(n-1)}} d^{N+1}(t) \mathbf{P}\left(X_{1^{t}}=k \mid X_{t}\right) .
\end{aligned}
$$

Since $T$ is a uniformly bounded tree, so $\left\{g_{t}(x, y)=\right.$ $\left.d^{N}(t) I_{k}(y), t \in T\right\}$ are uniformly bounded functions defined on $G^{2}$; then, from Lemma 3, we have

$$
\begin{aligned}
& \lim _{n \rightarrow \infty} \frac{S_{k}^{N}\left(T^{(n)}\right)-\sum_{t \in T^{(n-1)}} d^{N+1}(t) \mathbf{P}\left(X_{1^{t}}=k \mid X_{t}\right)}{\left|T^{(n+N)}\right|} \\
& \quad=0 \text { a.e. }
\end{aligned}
$$

Let $g_{t}(x, y)=d^{N+1}(t) \mathbf{P}\left(X_{1^{t}}=k \mid X_{t}=y\right)$ in Lemma 3; by Definition 1 and Lemma 4 , we have

$$
\begin{aligned}
E & {\left[g_{t}\left(X_{1_{t}}, X_{t}\right) \mid X_{1_{t}}\right] } \\
& =E\left[d^{N+1}(t) \mathbf{P}\left(X_{1^{t}}=k \mid X_{t}\right) \mid X_{1_{t}}\right] \\
& =d^{N+1}(t) E\left[E\left(I_{k}\left(X_{1^{t}}\right) \mid X_{t}\right) \mid X_{1_{t}}\right] \\
& =d^{N+1}(t) E\left[E\left(I_{k}\left(X_{1^{t}}\right) \mid \mathscr{F}_{|t|}\right) \mid \mathscr{F}_{|t|-1}\right] \\
& =d^{N+1}(t) E\left[I_{k}\left(X_{1^{t}}\right) \mid \mathscr{F}_{|t|-1}\right] \\
& =d^{N+1}(t) \mathbf{P}\left(X_{1^{t}}=k \mid X_{1_{t}}\right) .
\end{aligned}
$$


Since $\left\{g_{t}(x, y)=d^{N+1}(t) \mathbf{P}\left(X_{1^{t}}=k \mid X_{t}=y\right), t \in T\right\}$ are also uniformly bounded functions defined on $G^{2}$, from Lemma 3 and (18), for any $N \geq 0$, we have

$$
\begin{aligned}
\lim _{n \rightarrow \infty}( & \left(\sum_{t \in T^{(n-1)} \backslash\{0\}} d^{N+1}(t) \mathbf{P}\left(X_{1^{t}}=k \mid X_{t}\right)\right. \\
& \left.-\sum_{t \in T^{(n-1)} \backslash\{0\}} d^{N+1}(t) \mathbf{P}\left(X_{1^{t}}=k \mid X_{1_{t}}\right)\right) \\
\times & \left.\left(\left|T^{(n+N-1)}\right|\right)^{-1}\right)=0 \quad \text { a.e. }
\end{aligned}
$$

Hence,

$$
\begin{aligned}
\lim _{n \rightarrow \infty}( & \left(\sum_{t \in T^{(n-1)}} d^{N+1}(t) \mathbf{P}\left(X_{1^{t}}=k \mid X_{t}\right)\right. \\
& \left.-\sum_{t \in T^{(n-2)}} d^{N+2}(t) \mathbf{P}\left(X_{2^{t}}=k \mid X_{t}\right)\right) \\
\times & \left.\left(\left|T^{(n+N)}\right|\right)^{-1}\right)=0 \quad \text { a.e. }
\end{aligned}
$$

By (17) and (20), we have

$$
\begin{aligned}
\lim _{n \rightarrow \infty}( & \left(S_{k}^{N}\left(T^{(n)}\right)-\sum_{t \in T^{(n-2)}} d^{N+2}(t) \mathbf{P}\left(X_{2^{t}}=k \mid X_{t}\right)\right) \\
\times & \left.\times\left(\left|T^{(n+N)}\right|\right)^{-1}\right)=0 \text { a.e. }
\end{aligned}
$$

By induction, for fixed $N \geq 0$ and all $h \geq 1$, we have

$$
\begin{aligned}
& \lim _{n \rightarrow \infty} \frac{S_{k}^{N}\left(T^{(n)}\right)-\sum_{t \in T^{(n-h)}} d^{N+h}(t) \mathbf{P}\left(X_{h^{t}}=k \mid X_{t}\right)}{\left|T^{(n+N)}\right|} \\
& \quad=0 \text { a.e. }
\end{aligned}
$$

By (12), we have

$$
\begin{aligned}
& \sum_{t \in T^{(n-h)}} d^{N+h}(t) \mathbf{P}\left(X_{h^{t}}=k \mid X_{t}\right) \\
& \quad=\sum_{t \in T^{(n-h)}} \sum_{l \in G} d^{N+h}(t) I_{l}\left(X_{t}\right) \mathbf{P}\left(X_{h^{t}}=k \mid X_{t}=l\right) \\
& =\sum_{l \in G} \sum_{t \in T^{(n-h)}} d^{N+h}(t) I_{l}\left(X_{t}\right) P^{(h)}(k \mid l) \\
& =\sum_{l \in G} S_{l}^{N+h}\left(T^{(n-h)}\right) P^{(h)}(k \mid l) .
\end{aligned}
$$

As $h \leq n$,

$$
\sum_{l \in G} S_{l}^{N+h}\left(T^{(n-h)}\right)=\left|T^{(N+n)}\right|-\left|T^{(N+h-1)}\right|,
$$

we have as $h \leq n$

$$
\begin{aligned}
& \left|\frac{\sum_{t \in T^{(n-h)}} d^{N+h}(t) \mathbf{P}\left(X_{h^{t}}=k \mid X_{t}\right)}{\left|T^{(n+N)}\right|}-\pi(k)\right| \\
& \leq\left|\frac{\sum_{l \in G} S_{l}^{N+h}\left(T^{(n-h)}\right)\left[P^{(h)}(k \mid l)-\pi(k)\right]}{\left|T^{(n+N)}\right|}\right| \\
& \quad+\frac{\left|T^{(N+h-1)}\right|}{\left|T^{(n+N)}\right|} \\
& \leq \sup _{l \in G}\left|P^{(h)}(k \mid l)-\pi(k)\right|+\frac{\left|T^{(N+h-1)}\right|}{\left|T^{(n+N)}\right|} .
\end{aligned}
$$

Since $P$ is strongly ergodic, the first term of right-hand side of (25) is arbitrary small for large $h$, and the limit of second term is zero as $n \rightarrow \infty$; (15) can be obtained from (22) and (25).

Theorem 6. Under the conditions of Theorem 5 , let $S_{l, k}^{N}(A)$ be defined by (13); then

$$
\lim _{n \rightarrow \infty} \frac{S_{l, k}^{N}\left(T^{(n)} \backslash\{o\}\right)}{\left|T^{(n+N)}\right|}=\pi(l) P(k \mid l) \quad \text { a.e., } k, l \in G .
$$

Proof. Let $g_{t}(x, y)=d^{N}(t) I_{l}(x) I_{k}(y)$ for all $t \in T$ in Lemma 3 ; it is easy to see that $\left\{g_{t}(x, y), t \in T\right\}$ are uniformly functions defined in $G^{2}$, and, by (8) and Lemma 3, we have

$$
\begin{aligned}
H_{n}(\omega)= & \sum_{t \in T^{(n)} \backslash\{0\}} d^{N}(t) I_{l}\left(X_{1_{t}}\right) I_{k}\left(X_{t}\right)=S_{l, k}^{N}\left(T^{(n)} \backslash\{o\}\right), \\
G_{n}(\omega) & =\sum_{t \in T^{(n)} \backslash\{0\}} E\left[d^{N}(t) I_{l}\left(X_{1_{t}}\right) I_{k}\left(X_{t}\right) \mid X_{1_{t}}\right] \\
& =\sum_{t \in T^{(n)} \backslash\{0\}} I_{l}\left(X_{1_{t}}\right) d^{N}(t) \mathbf{P}\left(X_{t}=k \mid X_{1_{t}}=l\right) \\
& =\sum_{t \in T^{(n-1)}} d^{N+1}(t) I_{l}\left(X_{t}\right) P(k \mid l) \\
& =S_{l}^{N+1}\left(T^{(n-1)}\right) P(k \mid l),
\end{aligned}
$$$$
\lim _{n \rightarrow \infty} \frac{S_{l, k}^{N}\left(T^{(n)} \backslash\{o\}\right)-S_{l}^{N+1}\left(T^{(n-1)}\right) P(k \mid l)}{\left|T^{(n+N)}\right|}=0 \quad \text { a.e. }
$$

Equation (26) follows from (28) and Theorem 5.

Let $N=0$ in Theorems 5 and 6 ; we can obtain the strong law of large numbers for the frequencies of occurrence of states and ordered couples of states for countable Markov chains indexed by the uniformly bounded tree. 
Corollary 7. Under the conditions of Theorem 5, let

$$
\begin{aligned}
S_{k}\left(T^{(n)}\right) & =S_{k}^{0}\left(T^{(n)}\right)=\left|\left\{t \in T^{(n)}: X_{t}=k\right\}\right|, \\
S_{l, k}\left(T^{(n)} \backslash\{o\}\right) & =S_{l, k}^{0}\left(T^{(n)} \backslash\{o\}\right) \\
& =\left|\left\{t \in T^{(n)} \backslash\{o\}:\left(X_{1_{t}}, X_{t}\right)=(l, k)\right\}\right| ;
\end{aligned}
$$

then

$$
\begin{gathered}
\lim _{n \rightarrow \infty} \frac{S_{k}\left(T^{(n)}\right)}{\left|T^{(n)}\right|}=\pi(k) \quad \text { a.e., } k \in G, \\
\lim _{n \rightarrow \infty} \frac{S_{l, k}\left(T^{(n)} \backslash\{o\}\right)}{\left|T^{(n)}\right|}=\pi(l) P(k \mid l) \quad \text { a.e., } k, l \in G .
\end{gathered}
$$

Proof. Letting $N=0$ in Theorems 5 and 6, this corollary follows.

From Theorems 5 and 6, we can obtain easily the strong law of large numbers for countable Markov chains indexed by a Cayley tree [16] and the strong law of large numbers for finite Markov chains indexed by a uniformly bounded tree [15].

\section{Conflict of Interests}

The authors declare that there is no conflict of interests regarding the publication of this paper.

\section{Acknowledgments}

This work was supported by the National Natural Science Foundation of China 11071104, the Postgraduate Innovation Projection of Jiangsu University CXLX12-0652, Youth Foundation of Xuzhou Institute of Technology XKY2012301, National Natural Science Foundation of China 11226210, the Research Foundation for Advanced Talents of Jiangsu University 11JDG116, and the National Natural Science Foundation of China 11326174.

\section{References}

[1] I. Benjamini and Y. Peres, "Markov chains indexed by trees," The Annals of Probability, vol. 22, no. 1, pp. 219-243, 1994.

[2] D. Isaacson and R. Madsen, Markov Chains Theory and Application, John Wiley \& Sons, New York, NY, USA, 1976.

[3] C. E. Shannon, "A mathematical theory of communication," The Bell System Technical Journal, vol. 27, no. 3, pp. 379-423, 1948.

[4] B. McMillan, "The basic theorems of information theory," The Annals of Mathematical Statistics, vol. 24, no. 2, pp. 196-219, 1953.

[5] L. Breiman, "The individual ergodic theorem of information theory," The Annals of Mathematical Statistics, vol. 28, no. 3, pp. 809-811, 1957.

[6] L. Breiman, "Correction to the individual ergodic theorem of information theory," The Annals of Mathematical Statistics, vol. 31, no. 3, pp. 809-810, 1960.
[7] K. L. Chung, "A note on the ergodic theorem of information theory," The Annals of Mathematical Statistics, vol. 32, no. 2, pp. 612-614, 1961.

[8] J. Guyon, "Limit theorems for bifurcating Markov chains application to the detection of cellular aging," The Annals of Applied Probability, vol. 17, no. 5-6, pp. 1538-1569, 2007.

[9] T. Berger and Z. Ye, "Entropic aspects of random fields on trees," IEEE Transactions on Information Theory, vol. 36, no. 5, pp. 1006-1018, 1990.

[10] Z. Ye and T. Berger, "Ergodic, regulary and asymptotic equipartition property of random fields on trees," Journal of Combinatorics, Information \& System Sciences, vol. 21, no. 2, pp. 157-184, 1996.

[11] Z. Ye and T. Berger, Information Measures for Discrete Random Field, Science Press, Beijing, China, 1998.

[12] R. Pemantle, "Antomorphism invariant measure on trees," The Annals of Probability, vol. 20, no. 3, pp. 1549-1566, 1992.

[13] W. Yang, "Some limit properties for Markov chains indexed by a homogeneous tree," Statistics \& Probability Letters, vol. 65, no. 3, pp. 241-250, 2003.

[14] W. Yang and Z. Ye, “The asymptotic equipartition property for nonhomogeneous Markov chains indexed by a homogeneous tree," IEEE Transactions on Information Theory, vol. 53, no. 9, pp. 3275-3280, 2007.

[15] H. Huang and W. Yang, "Strong law of large numbers for Markov chains indexed by an infinite tree with uniformly bounded degree," Science in China A, vol. 51, no. 2, pp. 195-202, 2008.

[16] B. Wang, W. G. Yang, and Z. Y. Shi, "Strong laws of large numbers for countable Markov hains indexed by a Cayley tree," Scientia Sinica A, vol. 42, pp. 1031-1036, 2012 (Chinese). 


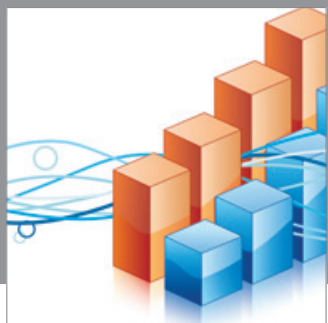

Advances in

Operations Research

mansans

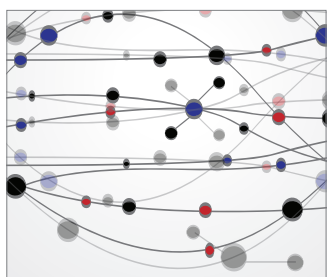

The Scientific World Journal
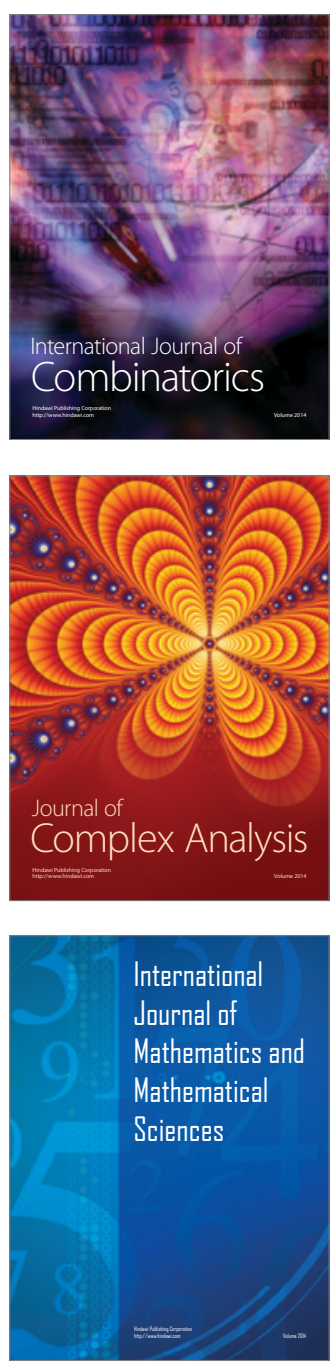
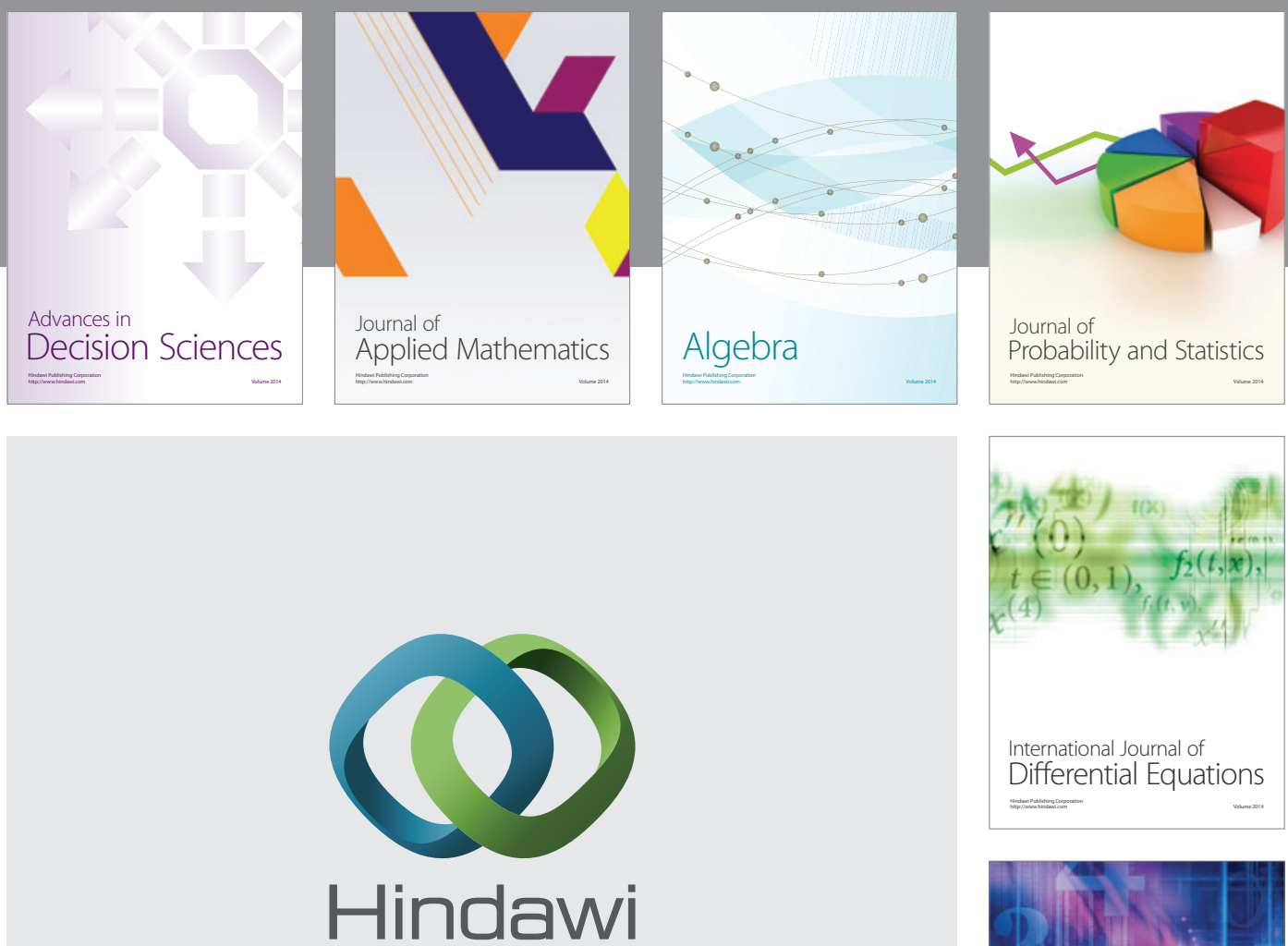

Submit your manuscripts at http://www.hindawi.com
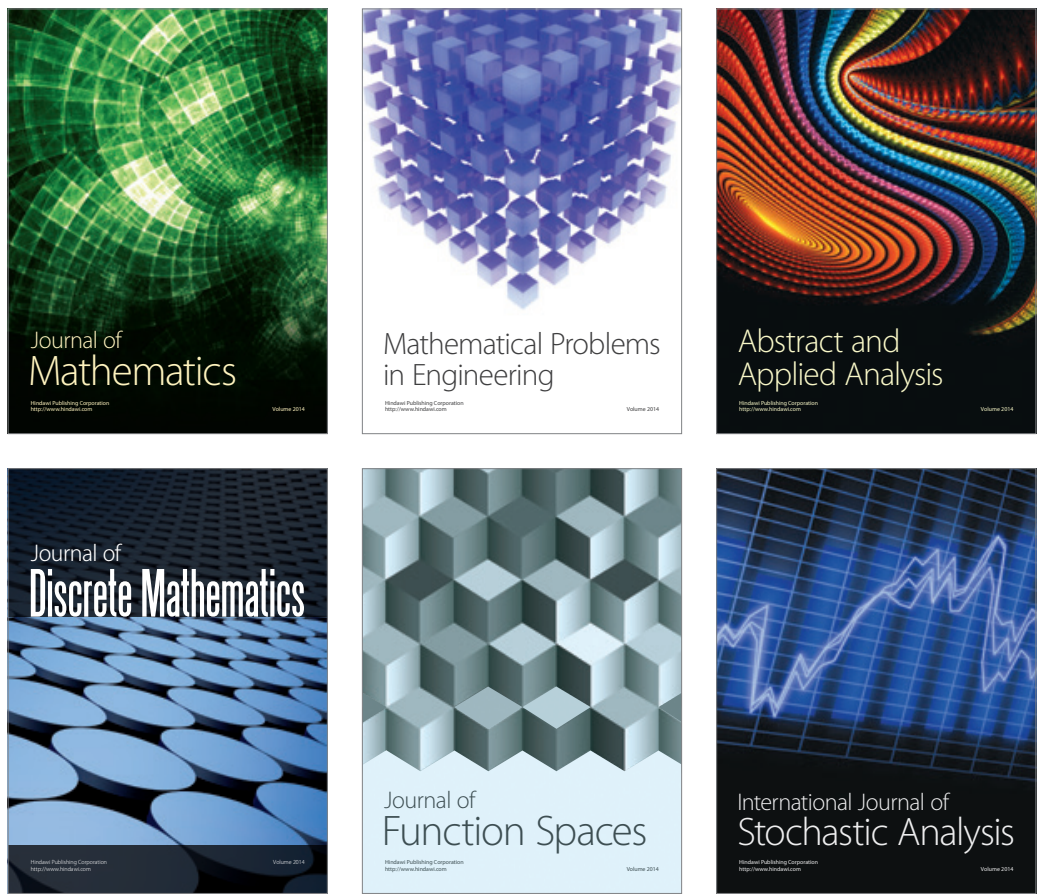

Journal of

Function Spaces

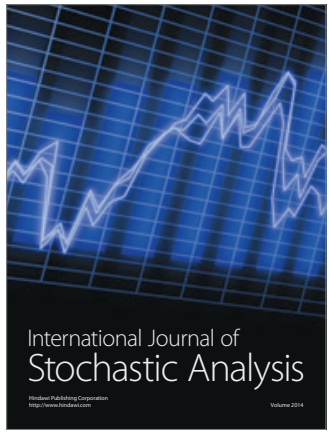

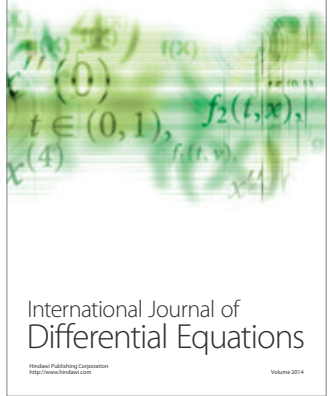
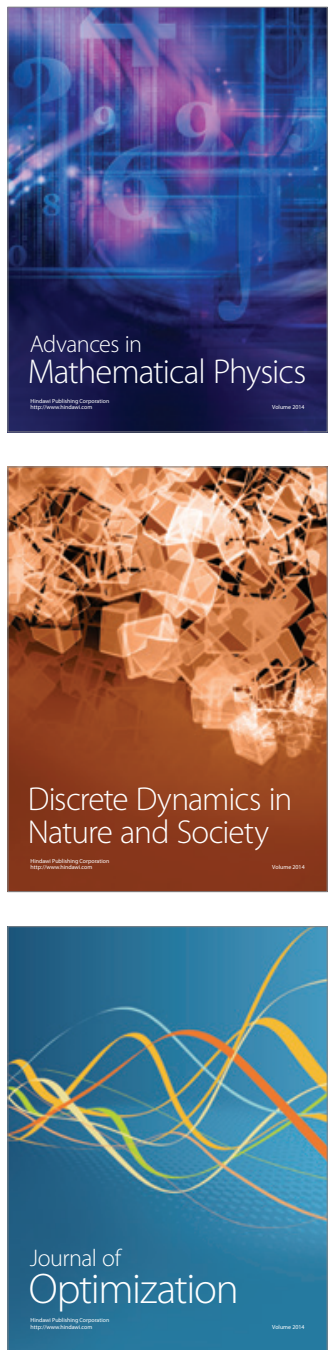